\title{
1 Geomagnetic field intensity as a cue for the regulation of insect
}

\section{2 migration}

3

4 Guijun Wan ${ }^{\mathrm{a}}$, Ruiying Liu ${ }^{\mathrm{a}}$, Chunxu $\mathrm{Li}^{\mathrm{a}}$, Jinglan $\mathrm{He}^{\mathrm{a}}$, Weidong Pan ${ }^{\mathrm{b}}$, Gregory A.

5 Sword ${ }^{\mathrm{c}}$, Gao Hu $\mathrm{Hu}^{\mathrm{a}}$, Fajun Chen ${ }^{\mathrm{a}, *}$

6

$7 \quad{ }^{a}$ Department of Entomology, College of Plant Protection, Nanjing Agricultural University, Nanjing 210095, China

$8{ }^{b}$ Beijing Key Laboratory of Bioelectromagetics, Institute of Electrical Engineering, Chinese Academy of Sciences,

$9 \quad$ Beijing 100190, China

$10{ }^{\mathrm{c}}$ Department of Entomology, Texas A\&M University, College Station, TX, U.S.A.

11

$12{ }^{*}$ Corresponding authors

13 E-mail addresses: fajunchen@njau.edu.cn (F.J. Chen)

14 


\section{Abstract}

2 Geomagnetic field (GMF) intensity can be used by some animals to determine their direction and

3 position during migration. However, its role, if any, in mediating other migration-related phenotypes

4 remains largely unknown. Here, we simulated variation in GMF intensity between two locations

5 along the migration route of a nocturnal insect migrant, the brown planthopper Nilaparvata lugens,

6 that varied by $\sim 5 \mu \mathrm{T}\left(\mathrm{GMF}_{50 \mu \mathrm{T}} \mathrm{vs} . \mathrm{GMF}_{45 \mu \mathrm{T}}\right)$ in field intensity. After one generation of exposure, we

7 tested for changes in key morphological, behavioural and physiological traits related to migratory

8 performance including wing dimorphism, flight capacity and positive phototaxis. Our results showed

9 that all three morphological and behavioural phenotypes responded to a small difference in magnetic

10 field intensity between the simulated northern vs. southern locations in ways expected along the

11 migratory route. Consistent magnetic responses in the expression of the phototaxis-related

12 Drosophila-like cryptochrome 1 (Cryl) gene and levels of two primary energy substrates used during

13 flight, triglyceride and trehalose, were also found. Our findings indicate GMF intensity can be a cue

14 that regulates the expression of phenotypes critical for insect migration and highlight the unique role

15 of magnetoreception as a trait that can help migratory insects express potentially beneficial

16 phenotypes in geographically variable environments.

17

18 Keywords

19 Geomagnetic field intensity; Brown planthopper; Magnetoreception; Wing dimorphism; Flight 20 capacity; Positive phototaxis 


\section{Introduction}

Organisms on Earth are perpetually surrounded by the geomagnetic field (GMF). Many animals have evolved the ability to sense or even utilize the GMF components of field intensity, direction, or both, although the details of the underlying biophysical mechanisms are still largely unknown $[1,2]$. Two mechanisms, including magnetite-based magnetoreception and radical-pair-based magnetoreception, have been studied most in terrestrial animals [3-5]. In particular, evidence is accumulating for radical-pair-based magnetoreception, which is mainly based on a quantum process involving different spin states of radical pairs under the effects of magnetic interactions and spin-selective reactions, with cryptochromes (Crys) as a potential agent for radical-pair-based magnetoreception [3, 4, 6-8].

In addition to using GMF for orientation and navigation, animals can also exhibit other physiological and behavioural responses to changes in magnetic field intensity [9-15]. However, few studies have focused on responses of animals to changes in geomagnetic field intensity at levels that naturally vary in their environment $[12,16]$. Most studies of the bioeffects of GMF on organisms in the last decade have been conducted by either shielding or enhancing the GMF, and have highlighted its importance in maintaining homeostasis $[13,15,17]$. Our work with migratory white-backed planthopper, Sogatella furcifera, showed that the absence of GMF can affect the expression of important migration-related behaviours, potentially through Cry-mediated hormone signaling. This includes changes in positive phototaxis which is critical for the ascent process, and flight capacity which is energetically-intensive and one of the main parameters used to assess migration ability [9, 18]. Additionally, previous work in migratory birds showed that changes in GMF information (both intensity and direction) over the migration route can affect their fuelling decisions and migratory restlessness [19-22]. As such, we speculated that changes in GMF intensity might play a more general underlying role in the regulation of insect migration.

Insects can conduct long-distance migrations in a short period of time [23, 24]. The nocturnal 
8

migratory brown planthopper, Nilaparvata lugens, has an annual long-distance migration in East Asia. Generally, their northward migration is initiated in spring from tropical and subtropical areas in Indochina to South China, followed by several subsequent waves of migration during the summer from South to North China, as well as to Japan and Korea Peninsula. Moreover, as they cannot overwinter in temperate East Asia, the insects then conduct a southward return migration through similar routes in the fall $[25,26]$. Physiological responses to variation in the GMF have been demonstrated in N. lugens [27]. Notably, both candidate magnetite crystals [28] and characterization of potential magnetic field targets, including Cry1, Cry2 [29], and iron-sulfur cluster assembly1 (IscA1) [30], have been systematically explored. Adult $N$. lugens also exhibit wing dimorphism associated with migration consisting of macropterous individuals with functional wings for seasonal migration and brachypterous individuals with vestigial wings and enhanced fecundity [31]. Given that outcomes and potential benefits of expressing specific migratory traits are predicted to change over the migratory route, we tested for changes in wing dimorphism, positive phototaxis, flight capacity, gene expression and flight physiology associated with variation in GMF intensity between the $N$. lugens emigration and immigration areas. We provide the first report of responses of migration-related traits to a magnetic field intensity change experienced by a long-distance migratory insect.

\section{Material and methods}

\section{Insect Stock}

Adult N. lugens were collected from paddy fields at Nanjing, Jiangsu province of China during their south-to-north migration in the summer of 2018 and reared indoors on susceptible Taichung Native 1 rice seedlings at $25 \pm 1 \square, 70-80 \% \mathrm{RH}$ and 14:10 h light: dark cycle (Dark during 1800-0800 hours) to establish a lab colony.

\section{Magnetic Fields and Insect Exposures}


1 The GMF intensity generally ranges from $\sim 25 \mu \mathrm{T}$ at the magnetic equator to $\sim 65 \mu \mathrm{T}$ at the magnetic

2 poles. We use two DC-type Helmholtz coil systems (External diameter: $1200 \mathrm{~mm}$ ) to mimic the

3 GMF intensity of two points on the migration route of $N$. lugens, Zhanjiang city $\left(21^{\circ} 12^{\prime} 29^{\prime \prime} \mathrm{N}, 110^{\circ}\right.$

$4 \quad 21^{\prime} 11^{\prime \prime} \mathrm{E}$; intensity: $\left.45000 \pm 255 \mathrm{nT}, \mathrm{GMF}_{45 \mu \mathrm{T}}\right)$ and Nanjing city $\left(32^{\circ} 3^{\prime} 42^{\prime \prime} \mathrm{N}, 118^{\circ} 46^{\prime} 40^{\prime \prime} \mathrm{E}\right.$;

5 intensity: $\left.50000 \pm 239 \mathrm{nT}, \mathrm{GMF}_{50 \mu \mathrm{T}}\right)$ at same declination $\left(-5.7 \pm 1.32^{\circ}\right)$ and inclination $(50.2 \pm$

$61.23^{\circ}$ ). The GMF was altered in effective homogeneous areas of $300 \times 300 \times 300 \mathrm{~mm}^{3}$ inside each

7 coil system. The parameters of the simulated magnetic field were monitored daily with a fluxgate

8 magnetometer (Model 191A, HONOR TOP Magnetoelectric Technology Co., Ltd., Qingdao, China;

9 sensitivity: $\pm 1 \mathrm{nT}$ ). To ensure uniform environmental factors, the two coils systems were located in

10 the same room separated by $6.5 \mathrm{~m}$ to avoid interference with each other. The position of tested $N$.

11 lugens within the effective areas of the two coil systems was randomly changed in the same way

12 daily. Following an established rearing protocol, rice planthoppers were exposed to the $\mathrm{GMF}_{50 \mu \mathrm{T}} \mathrm{vs.}$

$13 \mathrm{GMF}_{50 \mu \mathrm{T}}$ treatments for one generation from mated $\mathrm{F} 0$ females to newly emerged $\mathrm{F} 1$ adults that were

14 used in the assays [9]. We exposed $N$. lugens to the different GMF intensities for one generation

15 because the insects were expected to undergo at least one more long-distance migration after F0 16 generation.

\section{Wing dimorphism and behavioural assays}

18 Once the F1 adults emerged, individuals from each GMF group were identified to sex and wing form.

19 Knowing that only macropterous adults can migrate, and most migrants are unmated [32], macropterous unmated adults from the $\mathrm{GMF}_{50 \mu \mathrm{T}}$ and $\mathrm{GMF}_{45 \mu \mathrm{T}}$ groups were respectively tested for positive phototaxis, and flight capacity. According to the responses of the closely-related migratory species $S$. furcifera to GMF compensation, and given that 2-day-old adult $N$. lugens begin to take off for migration [25], newly emerged to 3-day-old adults (D1-D3) and 2-day-old (D2) adults were

24 investigated for positive phototaxis and flight capacity from 1800-0800 hours (subjective dark 25 period), respectively. Phototaxis was assessed using a previously developed assay system for rice 
1 planthoppers [9]. To ensure that the only difference between the groups was the imposed magnetic

2 field and considering that $N$. lugens would not all emerge on the same day, ten female or male adults

3 of the same post-emergence age (D1, D2 or D3) were randomly selected from $\mathrm{GMF}_{50 \mu \mathrm{T}}$ or GMF $\mathrm{GM}_{45 \mathrm{~T}}$

4 treatments and assigned to each channel of the phototaxis testing apparatus under corresponding

5 magnetic field conditions at 1745 hours (allowing $15 \mathrm{~min}$ for acclimation). The positive phototaxis

6 testing procedure used was the same as in [9] except that insects that failed to move to the light the

7 night before were not re-tested in this study. The final sample size for each D1-D3 test group was at

8 least 90 (see sample sizes in the figure legends). To compare flight distances between the two GMF

9 treatments, an infrared-beam based 8-channel flight mill system was constructed primarily with clear

10 acrylic plexiglass to avoid any anomalies triggered by ferromagnetic materials (granted patent:

11 ZL201620774681.1). The rice planthoppers could make a circular flight (diameter: $120 \mathrm{~mm}$ ) while

12 affixed to flight arm made from twisted copper wire (diameter: $2 \mathrm{~mm}$ ) with the vertical axis for the

13 attachment of the adult's with as previously described [9]. Eight insects could be tested per night.

14 An equal number of adults from each GMF treatment were randomly distributed to corresponding

15 flight mills at 1745 hours (allowing 15 min for acclimation). Any insects that appeared unhealthy or

escaped from their flight arms were excluded for further analyses. A total of fifteen 2-day-old adult $N$. lugens of each sex were tested in each GMF treatment group.

\section{Molecular and biochemical analysis}

We selected the multifunctional Crys (Cryl and Cry2) genes in 1 to 3-day-old macropterous unmated adults for transcript expression analysis by quantitative real-time polymerase chain reaction (qRT-PCR) using Arginine kinase and alpha 2-tubulin as reference genes. Nine biologically independent pools containing ten adults were frozen at the same time of 1800 hours in liquid nitrogen (collected at 1745 hours and acclimated for $15 \mathrm{~min}$ ) for each group divided by sex, magnetic field intensity and age, just before conducting the positive phototaxis experiment. Total RNA isolation, quality control, and concentration determination for total RNA, cDNA synthesis, and 
1 qRT-PCR were conducted as previously described [9]. Primer information can be found in the

2 supplementary material (table $\mathrm{S} 1$ ). The content of primary flight fuels, including triglyceride

3 (Jiancheng Bioengineering Institute, Nanjing, China) and trehalose (Suzhou Comin Biotechnology

4 Co. Ltd., Suzhou, China) [33, 34], in nine biologically independent pools containing ten 2-day-old

5 adults for each group frozen at the same time of 1800 hours in liquid nitrogen (collected at 1745

6 hours and acclimated for $15 \mathrm{~min}$ ) just before the flight capacity test, was also determined using

7 commercially available test kits.

\section{$8 \quad$ Statistical Analysis}

9 All data were analyzed using SPSS 20 (IBM Inc., Armonk, NY, U.S.A.). Associations among categorical variables were assessed with the two-sided Cochran-Mantel-Haenszel chi-square test, while continuous variables were analyzed with general linear models (GLM). For continuous variables, Shapiro-Wilk test was used to test for normality $(P>0.05)$ and Levene's test for the homogeneity of variances $(P>0.05)$, before an analysis of variance (ANOVA). In this study, all variables were separated by sex to investigate the main effect of GMF intensity. Therefore, we didn't take sex as a fixed factor in GLM, and no post-hoc multiple comparison tests for the sampling time or test day were performed. We used a two-sided Cochran-Mantel-Haenszel test to investigate the association between GMF intensity $\left(\mathrm{GMF}_{50 \mu \mathrm{T}}\right.$ or $\left.\mathrm{GMF}_{45 \mu \mathrm{T}}\right)$ and positive phototaxis stratified by test day (D1, D2, D3), and a two-way ANOVA to analyze the effects of the GMF intensity, sampling time (D1, D2, D3) and their interactions on gene expression levels of Cryl and Cry2 $(\log (\mathrm{x}+1)$ transformation) at $\alpha=0.05$ for both females and males. When we found a significant association between the two categorical variables, a follow-up chi-square test (two-tailed) with Yates's correction was performed to detail the association between GMF intensity and the positive phototaxis for females or males during the three test days. If a significant effect of GMF intensity or of interactions between the GMF intensity and sampling time was found on the gene expression levels, we used follow-up one-way ANOVA to compare the means for $\mathrm{GMF}_{50 \mu \mathrm{T}}$ and $\mathrm{GMF}_{45 \mu \mathrm{T}}$ at $\alpha=0.05$. A 
1 chi-square test (two-tailed) with Yates's correction was performed to investigate the association

2 between GMF intensity and wing dimorphism. A one-way ANOVA was used to test for the effect of

3 GMF intensity on flight distance, as well as the content of primary flight fuels for female or male

4 adults at $\alpha=0.05$, respectively. Effect sizes were estimated using Cohen's $w$ and partial $\eta^{2}$ for

5 chi-square test (small effect: $w=0.1$; medium effect: $w=0.3$; large effect: $w=0.5$ ) and ANOVA

6 (small effect: partial $\eta^{2}=0.01$; medium effect: partial $\eta^{2}=0.06$; large effect: partial $\eta^{2}=0.14$ ),

7 respectively, based on the benchmarks of Cohen (2013) [35].

\section{3. Results and discussion}

10 Exposure of $N$. lugens to variation in GMF intensities that mimicked those found at their emigration 11 and immigration locations affected the frequency of wing dimorphism. Significantly more adult 12 macropterous females $\left(128.57 \%, X_{1,323}^{2}=27.21, P<0.001, w=0.30\right)$ and males $\left(51.23 \%, X_{l}^{2}\right.$, $13367=17.24, P<0.001, w=0.22$ ) developed under $\mathrm{GMF}_{50 \mu \mathrm{T}} \mathrm{vs} . \mathrm{GMF}_{45 \mu \mathrm{T}}$ (figure $1 a$.). The observed 14 higher macropterous ratio, reflecting more migratory phenotype adults, would be expected in the 15 northern habitats that must be colonized by annual immigration and experience higher GMF 16 intensities relative to the overwintering site. This finding suggests that GMF intensity cues associated 17 with latitude can be used by $N$. lugens to help balance the trade-off between flight capability and 18 reproduction that is commonly observed in migratory animals [31]. For N. lugens, the relative 19 importance of reproductive capacity over flight should be higher at lower latitudes with lower GMF 20 intensity, because lower latitude areas serve as the overwintering site and source for the future 21 migrations [25]. 

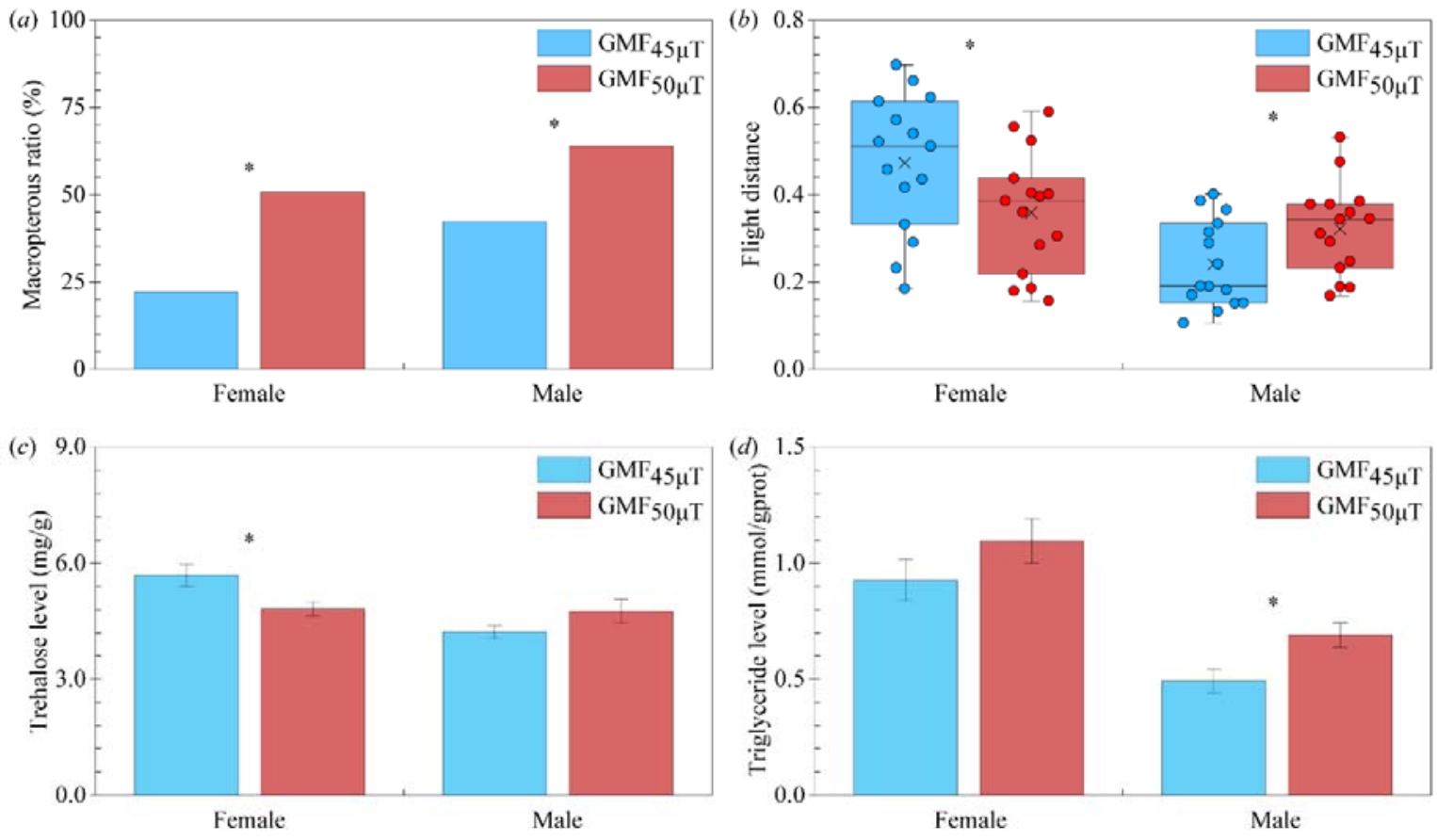

Figure 1. Wing dimorphism $(a)$, flight distance $(b)$, and main flight fuel trehalose $(c)$ and triglyceride (d) levels of Nilaparvata lugens under $\mathrm{GMF}_{50 \mu \mathrm{T}} \mathrm{vs} . \mathrm{GMF}_{45 \mu \mathrm{T}}$. In $(a), N=156$ and 167 for females, and 180 and 187 for males under $\mathrm{GMF}_{50 \mu \mathrm{T}} \mathrm{Vs} . \mathrm{GMF}_{45 \mu \mathrm{T}}$, respectively. In $(b), N=15$ for each group of unmated 2-day-old macropterous adults. In $(c)$ and $(d)$, samples of nine biologically independent pools containing ten unmated 2-day-old macropterous adults were used. $*$ denotes significant association between GMF intensity and wing dimorphism by chi-square test with Yates's correction $(a)$, and significant differences between $\mathrm{GMF}_{50 \mu \mathrm{T}} \mathrm{Vs} . \mathrm{GMF}_{45 \mu \mathrm{T}}$ by one-way $\mathrm{ANOVA}(b-d)$ for females or males at $p<0.05$.

Interestingly, the simulated $\sim 5 \mu \mathrm{T}$ GMF intensity change potentially caused by long-distance migration also revealed a sexually dimorphic effect on flight capacity of 2-day-old adult $N$. lugens. Higher $\mathrm{GMF}_{50 \mu \mathrm{T}}$ vs. $\mathrm{GMF}_{45 \mu \mathrm{T}}$ significantly extended male flight distance $\left(+33.84 \% ; F_{1,28}=4.74, P=\right.$ 0.038, partial $\left.\eta^{2}=0.15\right)$ whereas it shortened that of females $\left(-24.04 \% ; F_{1,28}=4.45, P=0.044\right.$, partial $\eta^{2}=0.14$ ) (figure $1 b$ ). As one of the most important performance parameters associated with insect migration, our data suggest that male $N$. lugens could make longer flights than female adults 
during the south-to-north emigration. One possible reason for this sexual dimorphism in dispersal ability could be to relieve intraspecific competition by avoiding competition with their progeny and

optimizing the use of resources. As main energy substances for insect flight, consistent significantly decreased trehalose level in females $\left(-15.18 \% ; F_{1,16}=5.89, P=0.027\right.$, partial $\eta^{2}=0.27$; figure $\left.1 c\right)$ and increased triglyceride level in males $\left(+40.46 \% ; F_{1,16}=6.33, P=0.023\right.$, partial $\eta^{2}=0.28$; figure 1d) were found under $\mathrm{GMF}_{50 \mu \mathrm{T}} \mathrm{vs} . \mathrm{GMF}_{45 \mu \mathrm{T}}$, indicating the potential pathway regulating trehalose and triglyceride (e.g., adipokinetic, insulin and juvenile hormone signaling) that is likely to be responsible for the sex-specific responses of flight distance to the GMF changes during migration[34, 36]. Taken together, the sexually dimorphic responses of flight capacity to the variation in the GMF likely reflects the different resource allocation strategies of females and males associated with long distance migration.

During the initial phase of migration, temporary inhibition of positive phototaxis was reported in $N$. lugens $[37,38]$. In the first three days after eclosion that we monitored, unmated macropterous adults showed significantly weakened positive phototaxis under $\mathrm{GMF}_{50 \mu \mathrm{T}}$ vs. $\mathrm{GMF}_{45 \mu \mathrm{T}}$ $(\mathrm{OR}=0.61,95 \%$ CI: $0.50-0.76, P<0.001, w=0.11)$. Compared with $\mathrm{GMF}_{45 \mu \mathrm{T}}$, the higher $\mathrm{GMF}_{50 \mu \mathrm{T}}$ that would be experienced at northern sites significantly decreased the percentage of unmated macropterous females and males that moved towards the light $(\lambda=320-680 \mathrm{~nm})$ by $30.39 \%\left(X_{1}^{2}\right.$, $660=7.05, P=0.008, w=0.11 ;$ figure $2 a)$ and $19.49 \%\left(X_{1,880}^{2}=7.92, P=0.005, w=0.10 ;\right.$ figure $\left.2 b\right)$ during the first 3 days, respectively. Specifically, we found that $\mathrm{GMF}_{50 \mu \mathrm{T}}$ significantly decreased the move-to-light ratios of unmated macropterous females on the 2nd $\left(-55.26 \% ; X^{2}{ }_{1}\right.$, $180=10.47, P=0.001, w=0.25)$ and 3 rd day $\left(-36.59 \% ; X_{1,220}^{2}=4.206, P=0.040, w=0.15\right.$; figure $2 a$ ), while males only on the 3 rd day $\left(-35.50 \% ; X_{1,250}^{2}=14.31, P<0.001, w=0.25\right.$; figure $\left.2 b\right)$. Although the biological significance of positive phototaxis is still an open question, it has been associated with migration in $N$. lugens $[37,38]$ and the weakened positive phototaxis under a higher GMF intensity as would be expected in northern locations may help adult $N$. lugens smoothly ascend 
1 to the sky after take-off for their each wave of south-to-north migration without distraction by light

2 sources on the ground [38].

3 Extreme variation in GMF exposure has previously been shown to affect wing dimorphism, flight

4 duration, and positive phototaxis in another rice planthopper species, S. furcifera [9]. However, the

5 changes observed were induced by exposure to near-zero GMF. Importantly, our results here for $N$.

6 lugens were induced by a biologically- and ecologically-relevant degree of variation in GMF levels

7 between southern regions that serve as the source of migrants and northern regions where

8 populations are re-established by migratory individuals every year. Thus, our results indicate that

9 naturally-occurring variation in GMF intensity can affect the expression of traits involved in

10 migration to result in phenotypes that favor different migratory and reproductive strategies in

11 different locations as part of the annual migration cycle.

12

13 Table 1. Two-way ANOVAs with the geomagnetic field (GMF) as the main factor and sampling day

14 as the sub-factor on the gene expression levels of cryptochromes (Cryl and Cry2) for the unmated

15 macropterous female and male adults.

\begin{tabular}{|c|c|c|c|c|c|c|c|}
\hline$\overline{S e x}$ & Genes & $d f$ & $\mathrm{GMF}^{\mathrm{a}}$ & $d f$ & Sampling day ${ }^{b}$ & $d f^{\mathfrak{c}}$ & GMF*Sampling day \\
\hline \multirow{3}{*}{ Female } & Cryl & 1,48 & $11.77 / 0.001(0.20)$ & 2,48 & $5.64 / 0.006(0.19)$ & 2,48 & $1.37 / 0.264(0.05)$ \\
\hline & & & & & & & \\
\hline & Cry 2 & 1,48 & $6.14 / 0.017(0.11)$ & 2,48 & $98.15 /<0.001(0.80)$ & 2,48 & $2.09 / 0.135(0.08)$ \\
\hline \multirow{2}{*}{ Male } & Cryl & 1,48 & $6.59 / 0.013(0.12)$ & 2,48 & $2.21 / 0.121(0.08)$ & 2,48 & $4.71 / 0.014(0.16)$ \\
\hline & Cry 2 & 1,48 & $9.24 / 0.004(0.16)$ & 2,48 & $108.50 /<0.001(0.82)$ & 2,48 & $7.35 / 0.002(0.23)$ \\
\hline
\end{tabular}

$16 \quad$ Results are given as $F$ value/ $P$ value (partial $\eta 2$ ).

$17{ }^{\mathrm{a}} \mathrm{GMF}-$ the $\mathrm{GMF}_{50 \mu \mathrm{T}}$ vs. $\mathrm{GMF}_{45 \mu \mathrm{T}}$.

$18{ }^{\mathrm{b}}$ Sampling day - 18:00 on the 1st (D1), 2nd (D2) and 3rd (D3) day after the adult emergence.

$19{ }^{\mathrm{c}}$ Ten individuals were randomly mixed as one sample. 

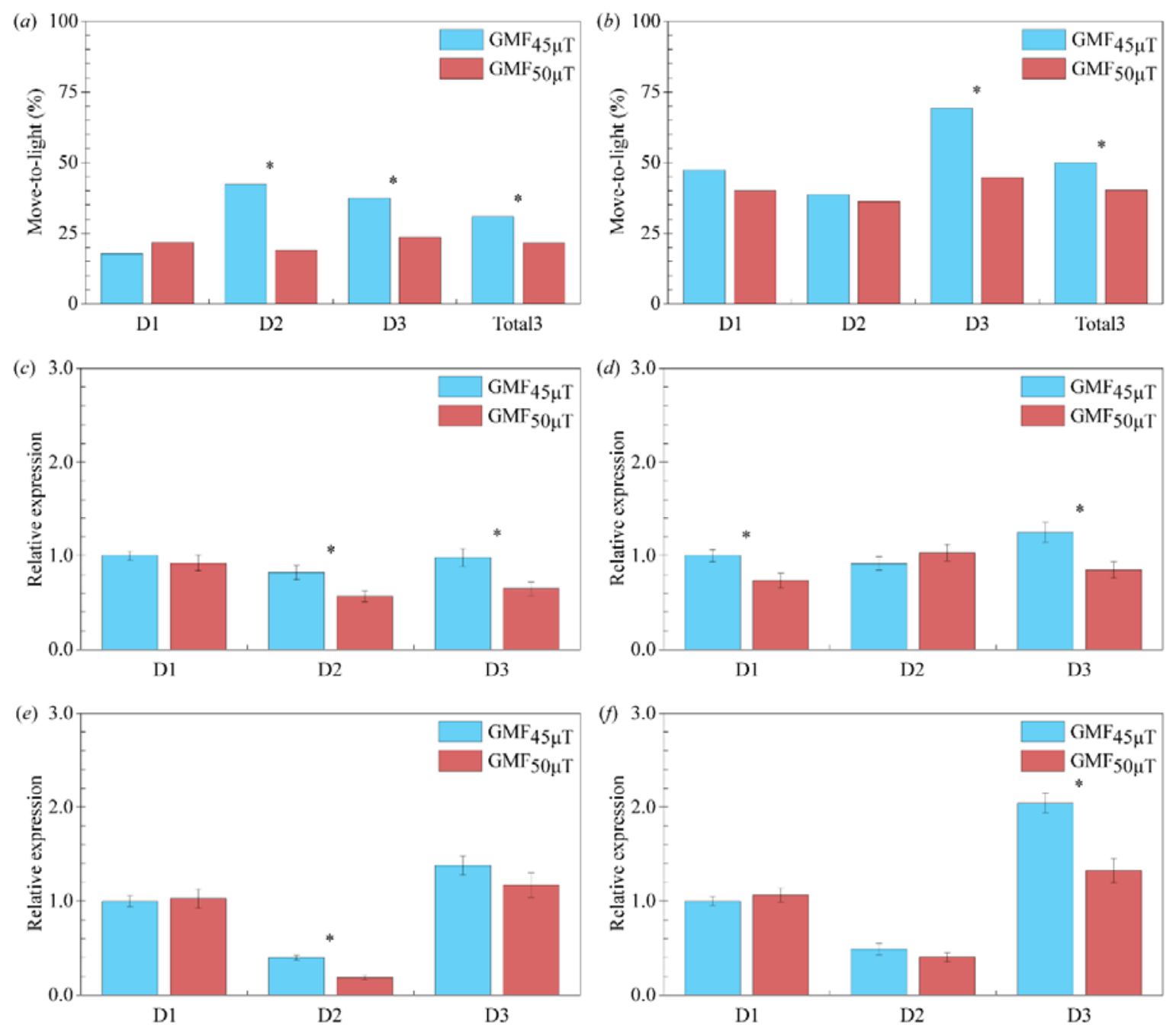

2 Figure 2. The positive phototaxis $(a, b)$ and transcript expression analyses for the multifunctional

3 cryptochromes (Cryl $(c, d)$ and Cry $2(e, f))$ of unmated macropterous female (left panels) and male

4 (right panels) Nilaparvata lugens under $\mathrm{GMF}_{50 \mu \mathrm{T}}$ vs. $\mathrm{GMF}_{45 \mu \mathrm{T}}$. For positive phototaxis, move-to-light

5 percentage of adults on the 1st-3rd day (D1-D3) and in the first three days (Total3) after emergence

6 were investigated. $N=130,90,110,330$, respectively, for the females in D1-D3 and Total3 under

7 both $\mathrm{GMF}_{50 \mu \mathrm{T}}$ and $\mathrm{GMF}_{45 \mu \mathrm{T}} ; N=130,130,130,390$, respectively, for the males in D1-D3 and Total3

8 under $\mathrm{GMF}_{50 \mu \mathrm{T}} ; N=220,150,120,490$, respectively, for the females in D1-D3 and Total3 under

$9 \mathrm{GMF}_{45 \mu \mathrm{T}}$. In $(c)-(f)$, samples of nine biologically independent pools containing ten unmated 10 macropterous adults were used. * denotes significant association between GMF intensity and 11 positive phototaxis by chi-square test with Yates's correction $(a, b)$, and significant differences 
between $\mathrm{GMF}_{50 \mu \mathrm{T}}$ vs. $\mathrm{GMF}_{45 \mu \mathrm{T}}$ by one-way ANOVA $(c-f)$ for each test (sampling) day at $p<0.05$.

It is well established that multifunctional cryptochromes are involved magnetoreception, phototaxis, and the circadian clock [39, 40], and developmental changes in gene expression of adult Cryl and Cry 2 associated with extreme variation in the GMF have been previously observed in $N$. lugens [29]. In our study, except for Cryl in males, the expression levels of Crys in both adult females and males on the 1st-3rd days (D1-D3) after emergence all varied significantly over time $\left(F_{2}\right.$, ${ }_{48} \geq 5.64, P \leq 0.006$, partial $\left.\eta^{2} \geq 0.19\right)$. Significant interactions between the GMF intensity and sampling day on the expression levels of Crys were only found in males $\left(F_{2,48} \geq 4.71, P \leq 0.014\right.$, partial $\eta^{2} \geq 0.16$ ), while the expression levels of Crys in both females and males were significantly affected by the GMF intensity $\left(F_{1,48} \geq 6.14, P \leq 0.017\right.$, partial $\eta^{2} \geq 0.11$; Table 1$)$. Mazzotta et al. (2013) found that the loss-of-function Drosophila Cry mutant can impair phototaxis of the fly [40]. Considering this, the consistent temporal expression pattern of Cryl may be responsible for the weakened positive phototaxis we observed. For both females and males, significantly decreased gene expression levels were observed in $2-\left(-30.78 \% ; F_{1,16}=6.10, P=0.025\right.$, partial $\left.\eta^{2}=0.28\right)$ and 3-day-old $\left(-34.09 \% ; F_{1,16}=7.00, P=0.018\right.$, partial $\eta^{2}=0.30$; figure $\left.2 c\right)$ females as well as 1 $\left(-26.67 \% ; F_{1,16}=6.19, P=0.024\right.$, partial $\left.\eta^{2}=0.28\right)$ and 3 -day-old $\left(-32.17 \% ; F_{1,16}=7.76, P=0.013\right.$, partial $\eta^{2}=0.33$; figure $2 d$ ) males under $\mathrm{GMF}_{50 \mu \mathrm{T}}$ vs. $\mathrm{GMF}_{45 \mu \mathrm{T}}$. Significantly down-regulated Cry2 expression was also found in 2-day-old females $\left(-52.86 \% ; F_{1,16}=29.36, P<0.001\right.$, partial $\eta^{2}=0.65$; figure $2 e)$ and 3-day-old males $\left(-35.24 \% ; F_{1,16}=16.75, P=0.001\right.$, partial $\eta^{2}=0.51$; figure $\left.2 f\right)$ adults. Unlike Drosophila-like Cry1, Vertebrate-like Cry2 has been reported to be vestigial flavoproteins [41] and would be unlikely to function as a magnetosensor by itself. Considering that Drosophila-like Cry1 is known to be involved in magnetosensing, it is possible in N. lugens Cry1 functions by itself for magnetoreception $[1,3,4]$. The magnetic response of positive phototaxis we found here is likely to be a result of interactions between the roles of Drosophila-like Cry1 in phototaxis and 
1 magnetoreception [1, 3, 4, 40], even though Cry2 may also function in a complex with

2 photoreceptors or downstream in magnetoreception signaling [42]. Moreover, given that Cry2 is a

3 component of the core circadian clock and it is reported that circadian clock is sensitive to changes in

4 magnetic field intensity [11, 43], a Cry2 function as a potential timing mechanism for migration

5 based on GMF intensity might also exist [44].

6

\section{4. Conclusion}

8 Taken together, our results provide the first evidence that the expression of crucial migration-related

9 traits such as wing dimorphism, flight capacity and phototaxis can respond to changes in local GMF

10 intensity during long-distance migration, indicating that the GMF can act as a cue for the regulation

11 of migratory phenotypes. $N$. lugens individuals seasonally migrate from their permanent southern

12 overwintering regions into higher latitude northern regions where overwinter survival is not possible.

13 Thus, behavioural, morphological and physiological changes that favor the development of more

14 mobile migratory phenotypes are predicted to be favored in northern latitudes to increase the

15 likelihood of successful return to the overwintering sites at the end of the season. Our results indicate

16 that $N$. lugens phenotypes can vary in response to changes in GMF in a manner consistent with the

17 expected trade-offs between reproduction and migration in permanent overwintering habitats in the

18 south and ephemeral habitats in the north colonized annually as part of the seasonal migration cycle.

19

\section{Acknowledgments}

21 We thank Likun Li for maintaining the flight mill and coils system, Jingyu Zhao and Jingjing Xu for 22 their useful discussion.

23

\section{$24 \quad$ Funding}

25 Funding was provided by the National Natural Science Foundation of China $(31701787,31470454$, 
131670855,51037006 and 31822043), the natural science foundation of Jiangsu Province

2 (BK20160717), the Fundamental Research Funds for the Central Universities (KJQN201820), the

3 Jiangsu Province Postdoctoral Science Foundation (1601196C), the Nanjing Agricultural University

4 Start-up Fund (82162045), and the National Basic Research Program of China (973)

5 (2010CB126200).

6

$7 \quad$ References

8 [1] Mouritsen, H. 2018 Long-distance navigation and magnetoreception in migratory animals. Nature 558, 50-59.

$9 \quad$ (doi:10.1038/s41586-018-0176-1).

10 [2] Chernetsov, N., Pakhomov, A., Kobylkov, D., Kishkinev, D., Holland, R.A. \& Mouritsen, H. 2017 Migratory

11 Eurasian reed warblers can use magnetic declination to solve the longitude problem. Curr. Biol. 27, 2647-2651.

12 (doi:10.1016/j.cub.2017.07.024).

13 [3] Hore, P.J. \& Mouritsen, H. 2016 The radical-pair mechanism of magnetoreception. Annu Rev Biophys 45,

14 299-344. (doi:10.1146/annurev-biophys-032116-094545).

15 [4] Ritz, T., Adem, S. \& Schulten, K. 2000 A model for photoreceptor-based magnetoreception in birds. Biophys. J.

16

17 [5] Kirschvink, J.L., Walker, M.M. \& Diebel, C.E. 2001 Magnetite-based magnetoreception. Curr. Opin. Neurobiol.

$18 \quad 11,462-467$.

19 [6] Maeda, K., Henbest, K.B., Cintolesi, F., Kuprov, I., Rodgers, C.T., Liddell, P.A., Gust, D., Timmel, C.R. \&

20 Hore, P.J. 2008 Chemical compass model of avian magnetoreception. Nature 453, 387-390.

21 (doi:10.1038/nature06834).

22 [7] Maeda, K., Robinson, A.J., Henbest, K.B., Hogben, H.J., Biskup, T., Ahmad, M., Schleicher, E., Weber, S.,

23 Timmel, C.R. \& Hore, P.J. 2012 Magnetically sensitive light-induced reactions in cryptochrome are consistent with

24 its proposed role as a magnetoreceptor. Proc. Natl. Acad. Sci. U.S.A. 109, 4774-4779. (doi:DOI

25 10.1073/pnas.1118959109).

26 [8] Liedvogel, M. \& Mouritsen, H. 2010 Cryptochromes--a potential magnetoreceptor: what do we know and what

27 do we want to know? J R Soc Interface 7 Suppl 2, S147-162. (doi:10.1098/rsif.2009.0411.focus).

28 [9] Wan, G., Yuan, R., Wang, W., Fu, K., Zhao, J., Jiang, S., Pan, W., Sword, G.A. \& Chen, F. 2016 Reduced 
1 geomagnetic field may affect positive phototaxis and flight capacity of a migratory rice planthopper. Anim Behav 121, 107-116. (doi:10.1016/j.anbehav.2016.08.024).

[10] Fedele, G., Green, E.W., Rosato, E. \& Kyriacou, C.P. 2014 An electromagnetic field disrupts negative geotaxis in Drosophila via a CRY-dependent pathway. Nat Commun 5, 4391. (doi:10.1038/ncomms5391).

[11] Yoshii, T., Ahmad, M. \& Helfrich-Forster, C. 2009 Cryptochrome mediates light-dependent magnetosensitivity of Drosophila's circadian clock. PLoS Biol. 7, e1000086. (doi:10.1371/journal.pbio.1000086).

[12] Bae, J.E., Bang, S., Min, S., Lee, S.H., Kwon, S.H., Lee, Y., Lee, Y.H., Chung, J. \& Chae, K.S. 2016 Positive geotactic behaviors induced by geomagnetic field in Drosophila. Mol Brain 9, 55. (doi:10.1186/s13041-016-0235-1).

[13] Binhi, V.N. \& Prato, F.S. 2017 Biological effects of the hypomagnetic field: an analytical review of experiments and theories. PLOS ONE 12, e0179340. (doi:10.1371/journal.pone.0179340).

[14] Roman, A. \& Tombarkiewicz, B. 2009 Prolonged weakening of the geomagnetic field (GMF) affects the immune system of rats. Bioelectromagnetics 30, 21-28. (doi:10.1002/bem.20435).

[15] Zhang, X., Yarema, K. \& Xu, A. 2017 Biological Effects of Static Magnetic Fields. Singapore, Springer.

[16] Lohmann, K.J. \& Lohmann, C.M.F. 1996 Detection of magnetic field intensity by sea turtles. Nature 380, 59-61. (doi:10.1038/380059a0).

[17] Choleris, E., Del Seppia, C., Thomas, A.W., Luschi, P., Ghione, G., Moran, G.R. \& Prato, F.S. 2002 Shielding, but not zeroing of the ambient magnetic field reduces stress-induced analgesia in mice. Proc. Biol. Sci. 269, 193-201. (doi:10.1098/rspb.2001.1866).

[18] Wan, G., Wang, W., Xu, J., Yang, Q., Dai, M., Zhang, F., Sword, G.A., Pan, W. \& Chen, F. 2015 Cryptochromes and hormone signal transduction under near-zero magnetic fields: new clues to magnetic field effects in a rice planthopper. PLOS ONE 10, e0132966. (doi:10.1371/journal.pone.0132966).

[19] Bulte, M., Heyers, D., Mouritsen, H. \& Bairlein, F. 2017 Geomagnetic information modulates nocturnal migratory restlessness but not fueling in a long distance migratory songbird. J. Avian Biol. 48, 75-82. (doi:10.1111/jav.01285).

[20] Fransson, T., Jakobsson, S., Johansson, P., Kullberg, C., Lind, J. \& Vallin, A. 2001 Bird migration: magnetic cues trigger extensive refuelling. Nature 414, 35-36 (doi:10.1038/35102115).

[21] Kullberg, C., Henshaw, I., Jakobsson, S., Johansson, P. \& Fransson, T. 2007 Fuelling decisions in migratory birds: geomagnetic cues override the seasonal effect. Proc. Biol. Sci. 274, 2145-2151. 
(doi:10.1098/rspb.2007.0554).

[22] Henshaw, I., Fransson, T., Jakobsson, S., Lind, J., Vallin, A. \& Kullberg, C. 2008 Food intake and fuel deposition in a migratory bird is affected by multiple as well as single-step changes in the magnetic field. J. Exp. Biol. 211, 649-653. (doi:10.1242/jeb.014183).

[23] Chapman, J.W., Reynolds, D.R. \& Wilson, K. 2015 Long-range seasonal migration in insects: mechanisms, evolutionary drivers and ecological consequences. Ecol. Lett. 18, 287-302. (doi:10.1111/ele.12407).

[24] Dreyer, D., Frost, B., Mouritsen, H., Gunther, A., Green, K., Whitehouse, M., Johnsen, S., Heinze, S. \& Warrant, E. 2018 The Earth's magnetic field and visual landmarks steer migratory flight behavior in the nocturnal Australian Bogong moth. Curr. Biol. 28, 2160-2166. (doi:10.1016/j.cub.2018.05.030).

[25] Cheng, X., WU, J. \& Ma, F. 2003 Brown planthopper: occurrence and control. Beijing, China, China Agricultural Press.

[26] Hu, G., Lu, M., Reynolds, D.R., Wang, H., Chen, X., Liu, W., Zhu, F., Wu, X., Xia, F., Xie, M., et al. 2019 Long-term seasonal forecasting of a major migrant insect pest: the brown planthopper in the Lower Yangtze River Valley. J Pest Sci (2004) 92, 417-428. (doi:10.1007/s10340-018-1022-9).

[27] Wan, G., Jiang, S., Zhao, Z., Xu, J., Tao, X., Sword, G.A., Gao, Y., Pan, W. \& Chen, F. 2014 Bio-effects of near-zero magnetic fields on the growth, development and reproduction of small brown planthopper, Laodelphax striatellus and brown planthopper, Nilaparvata lugens. J Insect Physiol 68, 7-15. (doi:10.1016/j.jinsphys.2014.06.016).

[28] Pan, W., Wan, G., Xu, J., Li, X., Liu, Y., Qi, L. \& Chen, F. 2016 Evidence for the presence of biogenic magnetic particles in the nocturnal migratory brown planthopper, Nilaparvata lugens. Sci Rep 6, 18771. (doi:10.1038/srep18771).

[29] Xu, J., Wan, G., Hu, D., He, J., Chen, F., Wang, X., Hua, H. \& Pan, W. 2016 Molecular characterization, tissue and developmental expression profiles of cryptochrome genes in wing dimorphic brown planthoppers, Nilaparvata lugens. Insect Sci. 23, 805-818. (doi:10.1111/1744-7917.12256).

[30] Xu, J., Zhang, Y., Wu, J., Wang, W., Li, Y., Wan, G., Chen, F., Sword, G.A. \& Pan, W. 2019 Molecular characterization, spatial-temporal expression and magnetic response patterns of iron-sulfur cluster assembly1 (IscA1) in the rice planthopper, Nilaparvata lugens. Insect Sci. 26, 413-423. (doi:10.1111/1744-7917.12546).

[31] Guerra, P.A. 2011 Evaluating the life-history trade-off between dispersal capability and reproduction in wing dimorphic insects: a meta-analysis. Biol Rev Camb Philos Soc 86, 813-835. 
(doi:10.1111/j.1469-185X.2010.00172.x).

[32] Zheng, D., Hu, G., Yang, F., Du, X., Yang, H., Zhang, G., Qi, G., Liang, Z., Zhang, X., Cheng, X., et al. 2014

Ovarian development status and population characteristics of Sogatella furcifera (Horváth) and Nilaparvata lugens (Stål): implications for pest forecasting. J. Appl. Entomol. 138, 67-77. (doi:10.1111/jen.12067).

[33] Zhao, Z. \& Zera, A.J. 2002 Differential lipid biosynthesis underlies a tradeoff between reproduction and flight capability in a wing-polymorphic cricket. Proc. Natl. Acad. Sci. U.S.A. 99, 16829-16834. (doi:10.1073/pnas.262533999).

[34] Arrese, E.L. \& Soulages, J.L. 2010 Insect fat body: energy, metabolism, and regulation. Annu. Rev. Entomol. 55, 207-225. (doi:10.1146/annurev-ento-112408-085356).

[35] Cohen, J. 2013 Statistical Power Analysis for the Behavioral Sciences. 2nd Edition ed. New York, Routledge. (doi:10.4324/9780203771587)

[36] Xu, J., Sheng, Z. \& Palli, S.R. 2013 Juvenile hormone and insulin regulate trehalose homeostasis in the red flour beetle, Tribolium castaneum. PLoS Genet. 9, e1003535. (doi:10.1371/journal.pgen.1003535).

[37] Hu, G., Lu, F., Lu, M., Liu, W., Xu, W., Jiang, X. \& Zhai, B. 2013 The influence of Typhoon Khanun on the return migration of Nilaparvata lugens (Stål) in eastern China. PLoS ONE 8, e57277. (doi:10.1371/journal.pone.0057277).

[38] Zhao, S. 1992 Behavioral mechanism of phototactic response of natural planthopper population (Sogatella furcifera and Nilaparvata lugens). Jiangxi Nongye Daxue Xuebao 4, 74-79.

[39] Chaves, I., Pokorny, R., Byrdin, M., Hoang, N., Ritz, T., Brettel, K., Essen, L.O., van der Horst, G.T., Batschauer, A. \& Ahmad, M. 2011 The cryptochromes: blue light photoreceptors in plants and animals. Annu Rev Plant Biol 62, 335-364. (doi:10.1146/annurev-arplant-042110-103759).

[40] Mazzotta, G., Rossi, A., Leonardi, E., Mason, M., Bertolucci, C., Caccin, L., Spolaore, B., Martin, A.J., Schlichting, M., Grebler, R., et al. 2013 Fly cryptochrome and the visual system. Proc. Natl. Acad. Sci. U.S.A. 110, 6163-6168. (doi:10.1073/pnas.1212317110).

[41] Kutta, R.J., Archipowa, N., Johannissen, L.O., Jones, A.R. \& Scrutton, N.S. 2017 Vertebrate cryptochromes are vestigial flavoproteins. Sci Rep 7, 44906. (doi:10.1038/srep44906).

[42] Bazalova, O., Kvicalova, M., Valkova, T., Slaby, P., Bartos, P., Netusil, R., Tomanova, K., Braeunig, P., Lee, H.J., Sauman, I., et al. 2016 Cryptochrome 2 mediates directional magnetoreception in cockroaches. Proc. Natl. Acad. Sci. U.S.A. 113, 1660-1665. (doi:10.1073/pnas.1518622113). 
1 [43] Zhang, Y., Markert, M.J., Groves, S.C., Hardin, P.E. \& Merlin, C. 2017 Vertebrate-like CRYPTOCHROME 2

2 from monarch regulates circadian transcription via independent repression of CLOCK and BMAL1 activity. Proc.

3 Natl. Acad. Sci. U.S.A. 114, E7516-E7525. (doi:10.1073/pnas.1702014114).

4 [44] Merlin, C. \& Liedvogel, M. 2019 The genetics and epigenetics of animal migration and orientation: birds,

5 butterflies and beyond. J. Exp. Biol. 222. (doi:10.1242/jeb.191890). 
